\title{
A Time-Domain Vibration Observer and Controller for Physical Human-Robot Interaction
}

This paper is a Post-Print version (ie final draft post-refereeing). For access to Publishers version, please access http://www.sciencedirect.com/science/article/pii/S0957415816300277.

Elsevier 2016.

\author{
Alexandre Lecours ${ }^{\mathrm{a}, *}$, Martin Otis ${ }^{\mathrm{b}}$, Pierre-Luc Belzile $^{\mathrm{a}}$, Clément Gosselin ${ }^{\mathrm{a}}$ \\ ${ }^{a}$ Département de génie mécanique, Université Laval, Québec, Canada, 1065 Avenue de la Médecine, G1V 0A6 \\ ${ }^{b}$ Département des sciences appliquées, Université du Québec à Chicoutimi, Saguenay, Québec, Canada
}

\begin{abstract}
This paper presents a time-domain vibration observer and controller for physical Human-Robot Interaction (pHRI). The proposed observer/controller aims at reducing or eliminating vibrations that may occur in stiff interactions. The vibration observer algorithm first detects minima and maxima of a given signal with robustness in regards to noise. Based on these extrema, a vibration index is computed and then used by an adaptive controller to adjust the control gains in order to reduce vibrations. The controller is activated only when the amplitude of the vibrations exceeds a given threshold and thus it does not influence the performance in normal operation. Also, the observer does not require a model and can analyze a wide time frame with only a few computations. Finally, the algorithm is implemented on two different prototypes that use an admittance controller.
\end{abstract}

Keywords: Physical Human-Robot Interaction, Vibration reduction, Admittance control, Intelligent assist device, Vibration control, Robotics

\section{Introduction}

Although robots have been used for several decades, direct physical interactions between robots and humans are rare, for obvious safety reasons. 5 The most evident means of ensuring safety is to segregate robots and human beings thereby leading to robots designed and programmed to work in a closed cell. However, in several applications, it is desirable to exploit the force capabilities of robots 10 by directly combining them with the skills of a human being, hence leading to human augmentation. The main challenge for human augmentation systems is to perceive their environment and the human intentions and to respond to them adequately, 15 intuitively and safely.

\footnotetext{
* Corresponding author

Email addresses: alexandre.lecours@gmc.ulaval.ca (Alexandre Lecours), martin_otis@uqac.ca (Martin Otis), clement.gosselin@gmc.ulaval.ca (Clément Gosselin)

Preprint submitted to Mechatronics
}

Physical human-robot interaction (pHRI) is emerging in many applications. In manufacturing, robots are used to work closely with operators in the same workspace. This includes for instance as20 sistive devices [1, 2] and new commercial robots such as the Kuka LWR 3 [3, Baxter 4, Universal Robots [5] and several more. In healthcare, a popular example is the da Vinci robotic surgical system used to assist surgeons. [6] 7]

The interaction with these devices must be safe and intuitive and a major concern to achieve this is related to stability and vibration issues. While the issue of stability is practically resolved in a large number of applications, vibrations remain a challenge, especially with stiff interactions.

It is well-known that the interaction between two different systems can generate vibrations in a closed-loop feedback scheme. This is especially true when the interaction is physically stiff. 8] Vibration problems arise from different sources, namely: limited bandwidth, latency or delay, discontinuities

September 27, 2016 
in the feedback loop or in the reference, modelling inaccuracies, mechanical friction, noise, sensor resolution and others.

40 This paper is structured as follows. First, a review of the state of the art in technologies for reducing vibration and analyzing system stability is presented. The primary contribution of this paper, namely, a non-linear algorithm for measuring and

45 eliminating vibrations coming from direct physical interaction is presented with the aim of improving operator safety. This algorithm is referred to in the following as an active time-domain vibration observer and controller. We then describe the 50 method used to extract a vibration index based on minima and maxima (extrema) from a given signal with robustness in regards to noise. This index is then used by an adaptive controller in order to reduce or eliminate the vibrations. The observer

55 does not require a model and can analyze a wide time frame with only a few computations. Also, the controller is activated only when the amplitude of the vibrations exceeds a given threshold (both in frequency and amplitude) and thus it does not influence the performance in normal operation. Finally, the implementation of the algorithm on two different prototypes is described in order to demonstrate its performance.

\section{Literature review}

Stability and vibration issues in haptics and pHRI have received considerable attention in the literature. A popular method to reduce vibrations is to use an artificial impedance or admittance link between the haptic display and a virtual world. The 70 objective is to decouple the haptic control and the model of the virtual environment 9 . This virtual coupling is always used and reduces the task performance. Another approach is to model the system and adaptively adjust the controller parame-

75 ters with the help of the sensors related to the human movement in order to avoid vibrations. The parameters can be adjusted using the well-known Routh-Hurwitz criterion, root-locus, Nyquist, Lyapunov, $\mu$-analysis, or other similar techniques [10].

A very popular method is to use the energy transferred in the system with concepts such as time domain passivity [11, 12, or absolute passivity 13. Passivity theory in the time domain has been used in many applications such as bilateral control of teleoperators under time-varying communication delays [14, 15] and for the control of haptic interfaces 16. In the latter case, virtual damping parameters are used to reduce vibrations. These passivity observer and controller (PO/PC) are only activated when required and thus they minimally degrade the performance 12 . Passivity theory is also applied in the frequency domain to adjust impedance filter parameters [17. and to define passivity-equivalent systems [18. Another frequency-domain stability observer is proposed in 19. One of the challenges of frequencydomain methods is the computational burden, especially with large data sets and real-time control constraints.

\section{Vibration observer}

This section explains the vibration observer/controller algorithm. The general principle is first presented, followed by the description of a wide time window and a narrow time window. Finally, the vibration index is defined. Figure 1 presents the general vibration observer-controller scheme while Fig. 2 presents the structure of the vibration observer. A video https://youtu.be/_VZMEmertCo (see Electronic 110 Annex 1 in the online version of this article) accompanying this paper summarizes the algorithms and shows experimental results [The video was placed on Youtube for the Review only].

\subsection{Proposed algorithm}

In order to assess the vibrations in a signal at a given time $t_{0}$, the last discrete $w$ points of the signal are considered. In terms of time, this is equivalent to the interval $t \in\left[\left(t_{0}-T_{w}\right), t_{0}\right]$, referred to as the wide time window. In the context of humanrobot interaction, the signal whose vibrations are analyzed can be the measured velocity, the desired velocity or the interaction force. The first step is to find all the minima and maxima of the signal in the wide time window. This is done by using a narrow time window technique. Based on these extrema, a vibration index is computed and is then used by an adaptive controller to adjust control gains thus reducing vibrations.

\subsection{Wide time window}

The duration of the wide time window, $T_{w}$, is a very important design parameter. Since the vibration index is based on the detection of minima and 


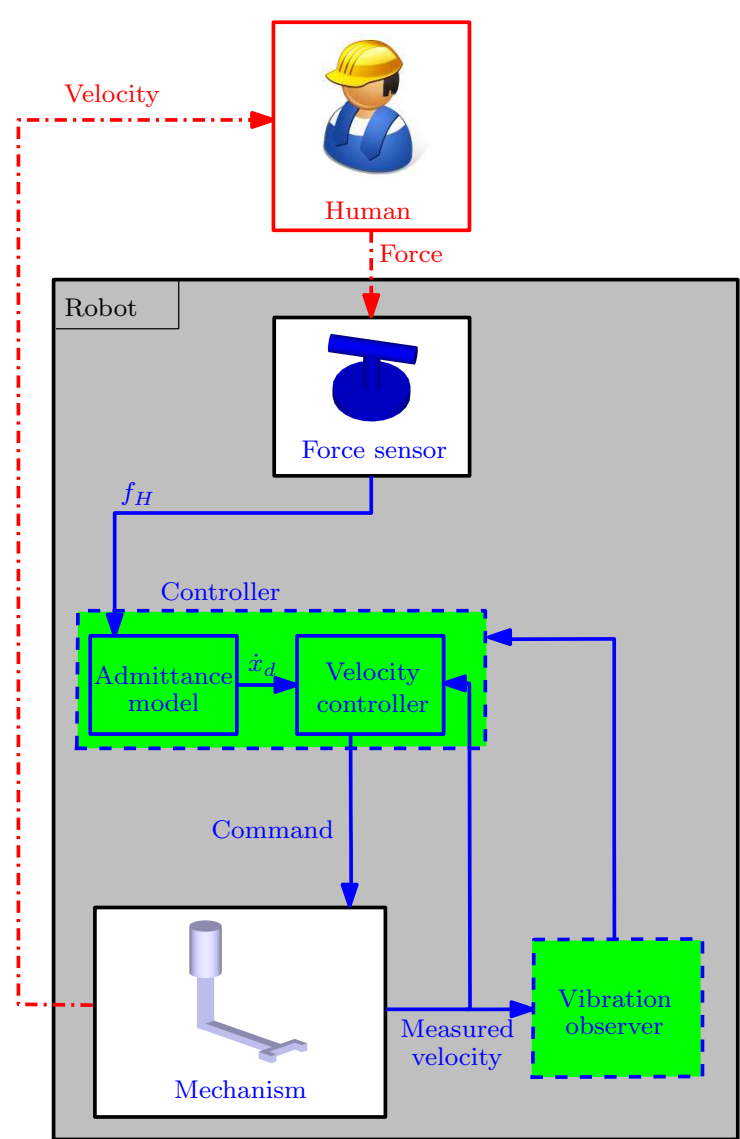

Figure 1: General structure of the vibration observercontroller: $r$ is the reference, $u$ is the control output, $y_{2}$ is the output and $y_{1}$ is the signal considered for the vibration observer.

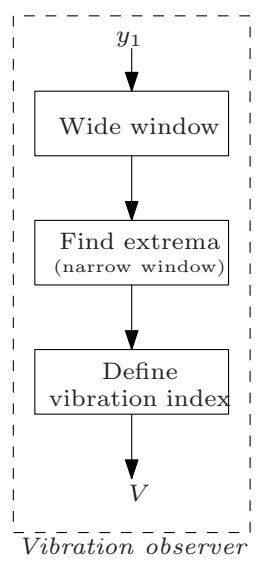

Figure 2: Structure of the vibration observer: $y_{1}$ is the observer input and $V$ is the observer ouput.

maxima, one should have

$$
T_{w}>\frac{1}{f_{l}}
$$

where $f_{l}$ is the lowest frequency to be accounted for in the vibration index. At a given time, only the vibrations inside the wide time window are considered. Figure 3 illustrates the concept of wide time window. The width of the wide time window may be adjusted depending on the applications. The authors suggest a minimum time frame of

$$
T_{w} \simeq \frac{3}{f_{l}}
$$

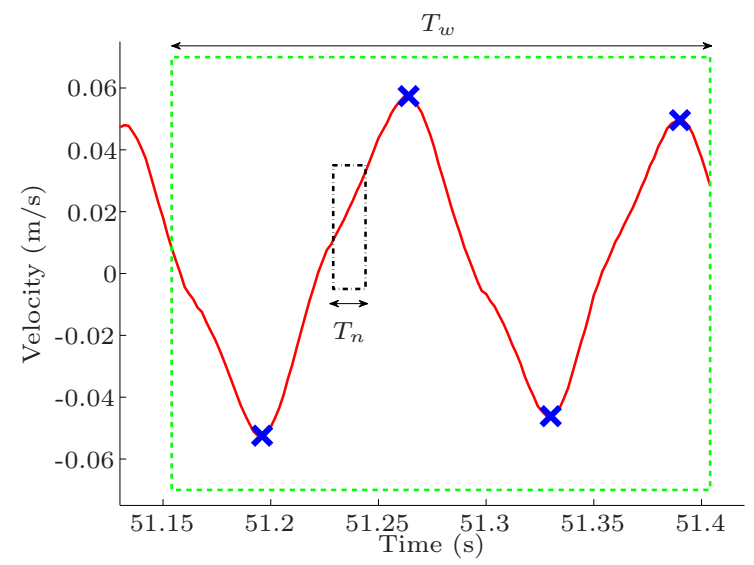

Figure 3: Illustration of the wide and narrow time windows: the green dashed lines represent the boundaries of the wide time window while black dashed-dot lines represent the boundaries of a narrow time window at a given point in the wide time window.

130

135

140

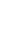
row time window is centred on the point to be tested and this point is registered if it is a maximum or minimum within the narrow time window.

The duration of the narrow time window $T_{n}$ is also an important design parameter. If it is small, a 
large number of minima and maxima will be found due to signal noise. On the other hand, if it is too large, high frequency vibrations will not be detected. Indeed, one has

$$
T_{n}<\frac{1}{2 f_{h}}
$$

where $f_{h}$ is the highest frequency to be accounted for in the vibration index. The choice of $T_{n}$ is largely dependent on the signal noise.

Figure 3 shows a wide-time window and a narrow time window. Figure 4 shows the minima and maxima obtained with a low-noise signal while Fig. 5 shows the results obtained with a noisy signal, demonstrating the algorithm's robustness to noise.

In mathematical terms, the signal is discretized using a sampling period $T_{s}$, which leads to $j=\frac{T_{n}}{T_{s}}+$ 1 signal samples, noted $s_{i}, i=1, \ldots, j$, where $s_{i}$ is the magnitude of the signal corresponding to the $i$ th sample. The magnitude of the signal for the sample corresponding to the centre of the narrow window, noted $s_{c}$, with $c=\frac{T_{n}}{2 T_{s}}$ is then considered. If one has

$$
s_{c}=\max \left(s_{1}, \ldots, s_{j}\right)
$$

then a maximum is detected while if

$$
s_{c}=\min \left(s_{1}, \ldots, s_{j}\right)
$$

a minimum is detected. Otherwise, no extremum is detected. Finally, if both a maximum and a minimum are detected for a given point, the latter is ignored. This narrow time window test is performed for each point in the wide time window.

It should be pointed out that the narrow time window does not scan the wide time window at each time step. Instead, it is possible to use the results from the preceding wide time window to reduce computational costs.

At this point in the algorithm, a series of minima and maxima of a given signal have been obtained with robustness in regards to noise.

\subsection{Vibration index}

Based on the minima and maxima detected in a given wide time window, a vibration index $(V)$ is proposed. First, the total number of extrema, noted $q$, is computed. If there are fewer than two extrema, the index is set to zero. Otherwise, the following definition is proposed for the vibration index:

$$
V=\lambda \sum_{i=1}^{q-1} \frac{\left|y_{1, i+1}-y_{1, i}\right|}{\left(t_{1, i+1}-t_{1, i}\right)}
$$

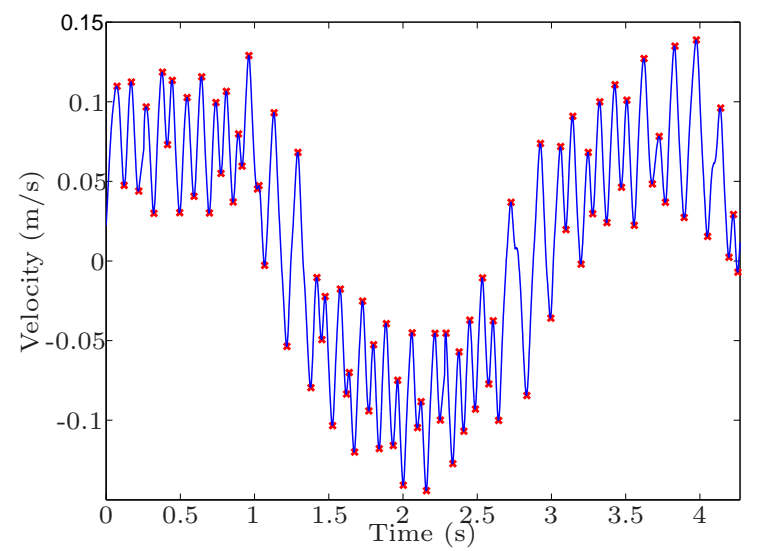

Figure 4: Minima and maxima detected by the algorithm for a low-noise signal $\left(T_{n}=0.03 \mathrm{~s}\right)$.

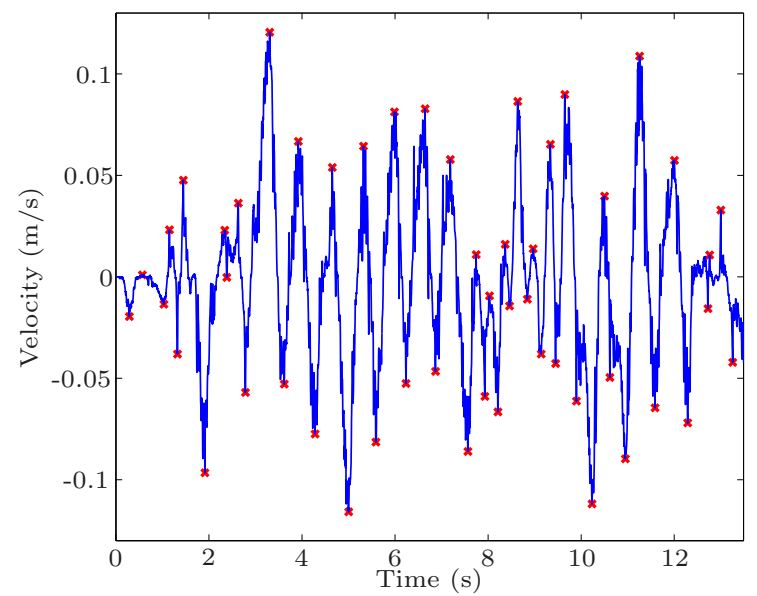

Figure 5: Minima and maxima detected by the algorithm for a noisy signal $\left(T_{n}=0.3 s\right)$. 
where $y_{1, i}$ and $t_{1, i}$ are respectively the signal amplitude and the time corresponding to the $i$ th ex-

175 tremum and $\lambda$ is a scaling factor. The latter is only used to scale the index, for simplicity of use.

The larger the difference between two consecutive extrema, the larger the index will be. Similarly, the shorter the time between two consecutive extrema, the larger the index will be. In other words, an increase in vibration amplitude or frequency results in a higher vibration index.

A modified version of the index may include limits on the index's rate of change. A rising limit $\left(L_{r}\right)$ and a falling limit $\left(L_{f}\right)$ are used to smooth the vibration index in order to avoid abrupt variations. A saturation can also be applied to prevent the vibration index from increasing indefinitely.

Figure 6 shows an example of the determination of the vibration index. On the left hand side, the signal is taken from a smooth human-robot interaction situation while on the right hand side the signal is taken from a stiff interaction, leading to 200 vibrations.
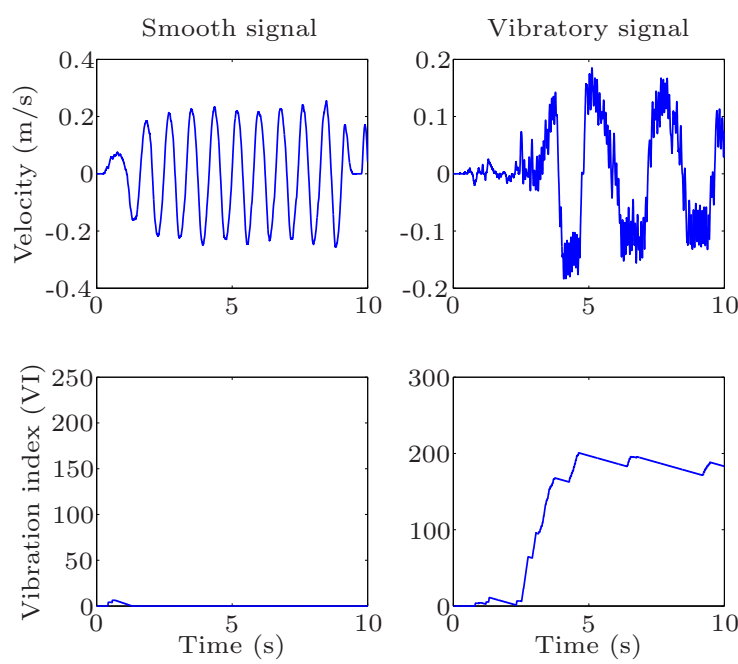

Figure 6: Example of the determination of the vibration in- 210 $\operatorname{dex}, V$, for two signals taken from a human-robot interaction experiment. On the left hand side with a smooth interaction situation and on the right hand side with a stiff interaction leading to vibrations $\left(T_{w}=0.25 \mathrm{~s}, T_{n}=0.03 \mathrm{~s}, \lambda=20\right.$, $\left.L_{r}=12.5 s^{-1}, L_{f}=0.5 s^{-1}\right)$

\section{Application to admittance control}

This section first recalls the concept of admit- 220 tance control and then presents the implementation of the vibration observer-controller.

\subsection{Admittance control}

Two main control classes are used in haptics and pHRI namely, impedance and admittance control 20]. Impedance controllers accept a displacement as input, which is measured, and react with a force. Devices controlled by this method should ideally have low inertia and friction since the user will inevitably feel these forces if they are not adequately compensated for. Admittance controllers, on the other hand, accept a force as input, which is measured, and react with a displacement [21, 22, 23]. Applications involving moderately large payloads usually use admittance control where a handle or a force/torque sensor is normally used to detect human intentions [24, 2, 23. Admittance control is detailed in [23, 25, 1]. The onedimensional admittance equation is written as:

$$
f_{H}=m \ddot{x}+c \dot{x} .
$$

where $f_{H}$ is the interaction force, i.e., the force applied by the human operator, $m$ the virtual mass, $c$ the virtual damping and $x, \dot{x}, \ddot{x}$ are respectively the position, velocity and acceleration.

The trajectory to be followed by the robot can be prescribed as a position $x_{d}$ or as a desired velocity $\dot{x}_{d}$. For velocity control, the desired velocity can be written, in the Laplace domain, as:

$$
\dot{X}_{d}(s)=\frac{F_{H}(s)}{m s+c}=\frac{F_{H}(s) / c}{\frac{m}{c} s+1}=F_{H}(s) H(s) .
$$

where $\dot{X}_{d}(s)$ is the Laplace transform of $\dot{x}_{d}, F_{H}(s)$ is the Laplace transform of $f_{h}$ and $s$ is the Laplace variable. Velocity control is used here, similarly to what was done in $[26,27,23$.

\subsection{Admittance control with vibration observer- controller}

Although stability issues pertaining to impedance control have been vastly explored [28, 29, 30, 31, not much has been reported for admittance control 32, 33.

With admittance control, vibrations or instability may occur when facing a stiff environment (in many cases the operator can be the source of stiffness.). In order to prevent such a situation - because safety is the primary concern - admittance parameters are then normally set to very conservative values. Therefore, large operator forces are required to move the device even under normal conditions. 
The objective here is to set the admittance parameters to low values in order to be able to easily move the device. The vibrations generated from a 2 stiff environment can be eliminated by the vibration observer/controller. There is still a trade-off between vibration and performance but the vibration controller is activated only when necessary and thus the trade-off is limited to exceptional situations.

\section{Vibration controller}

Once the level of vibrations are detected, the controller must be adapted in order to decrease or eliminate the vibrations. The following section presents adaptive control strategies to achieve this goal along with simulation results. The one degree-of-freedom (dof) system used in the simulations is shown in Fig. 7] where $m$ and $c$ are the admittance virtual mass and virtual damping, $f_{H}$ is the human force input, $v_{d}$ is the desired velocity and $v$ is the device velocity. The robot is represented as an inertia $\left(M_{r}\right)$ with damping $\left(C_{r}\right)$ and a PD controller is used, with parameters $K$ and $T_{d}$.

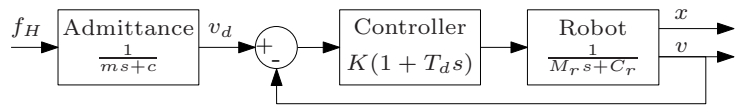

Figure 7: System used for the simulations.

\subsection{Adaptive control strategies}

Different control strategies are possible. In the human-robot interaction context, it is proposed to adapt the admittance parameters, to add damping or to modify the PID controller gain. Each of these cases are described below.

\subsubsection{Adaptive admittance parameters}

Referring to the admittance equation, i.e., eqn. (7), if the admittance parameters ( $m$ and $c$ ) are low, the robot is easy to move but is also be more reactive to high-frequency inputs (for instance when interacting with a stiff environment). If the parameters are high, the robot is more difficult to move but also less reactive to high frequency inputs. In order to be robust to high frequency inputs and be- 295 cause safety is the primary concern, the admittance parameters are normally set very high, thus requiring large forces to move the robot. A main objective of this research is to eliminate this compromise.

One possible approach is to set low admittance parameters in normal operation and to increase the parameters when vibrations are detected. The virtual mass and virtual damping must remain positive in order for the admittance transfer function to remain stable and the parameters should not be modified too quickly to avoid exciting the system.

A force profile shown in Fig. 8 is sent to the system as input. The first part $(0-13 s)$ is a low frequency sinusoidal signal, the second part is a constant input $(13-20 s)$, the third part is a high frequency sinusoidal signal $(20-30 s)$ and the last part is a constant input $(>30 s)$.

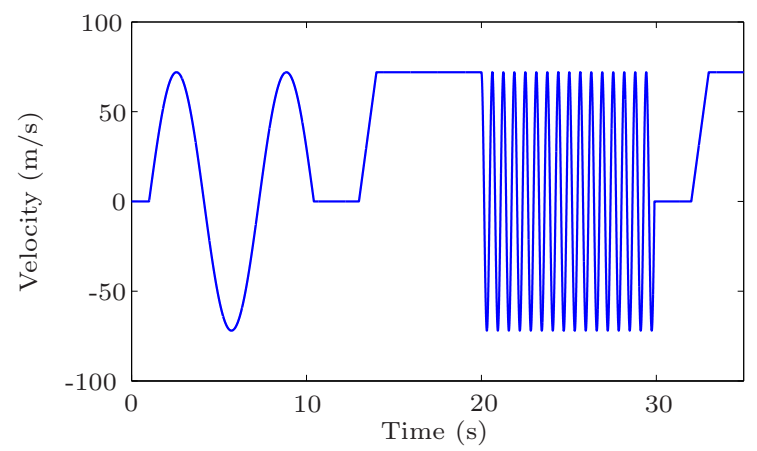

Figure 8: Force input profile used in the simulations.

Figure 9 shows the resulting desired velocity with different admittance parameters as well as the resulting vibration index. With low admittance parameters $(m=18, c=18)$, the system reaches high

280 puts. With high admittance parameters $(m=180$, $c=180)$, the system only reaches small velocities and would require large forces to move but it is not very reactive to high-frequency inputs. With the vibration controller, the system reaches high velocities and is not very reactive to high-frequency inputs. The parameters are varied linearly between $[m=18, c=18]$ (when the index $V$ is below 15 $\left(V_{\text {min }}\right)$ ) and $[m=180, c=180]$ (when the index $V$ is over $\left.25\left(V_{\max }\right)\right)$.

\subsection{Adaptive damping}

A common strategy to decrease vibrations is to add damping to the system. The principle is very simple and system stability is also easy to prove. However, there may be a practical limit of damping that can be added due to signal noise and communication delay. Since damping uses a signal derivative, a special attention in the selection of the differentiation algorithm is important in order to avoid 

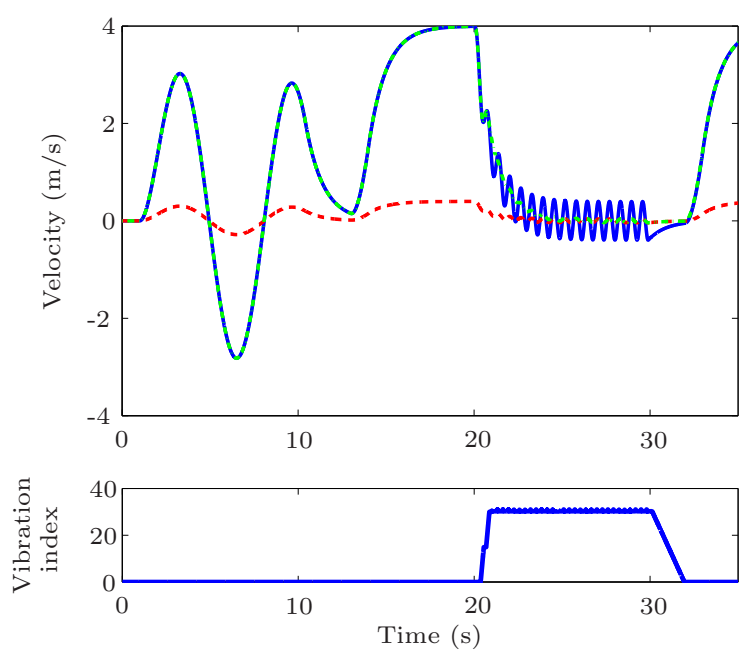

Figure 9: Desired velocity resulting from the force input shown in Fig. 8. The solid blue line is obtained with low admittance parameters $(m=18, c=18)$. The red dashed line is obtained with high admittance parameters $(m=180$, $c=180)$. The green dashed-dot line is obtained with the vibration controller (the parameters are varied between $(m=18, c=18)$ and $(m=180, c=180))$. The second subplot shows the vibration index.

noise amplification, which can increase the vibrations when the noise is transmitted to the actuators 34.

\subsection{Adaptive controller}

When faced with a stiff environment, robotic systems using admittance control tend to exhibit more vibrations when high loop gains are used. The control parameters can be set to lower values, but, as a compromise, the position or velocity would not follow the command as precisely. The objective here is to set high default controller gains but to decrease the controller gains when vibrations are detected. A first advantage of this method over modifying the admittance parameters is that it is more general and it can be applied in a variety of situations. A second advantage is that modifying 350 the admittance parameters may not work in some specific cases. For example, if there is a perturbation on the output leading to vibrations (severe noise or sensor error), lowering the controller gains would help but modifying the admittance parameters would not since it would only modify the reference.

The closed-loop transfer function of the system 355 (see Fig. 7) can be written as:

$$
\frac{v}{v_{d}}=\frac{K\left(1+T_{d} s\right)}{s\left(M+K T_{d}\right)+(K+C)} .
$$

Therefore, the state velocity can be written as

$$
v_{s}=S_{r} \cdot v_{d}=\frac{K}{K+C} \cdot v_{d}
$$

where $S_{r}$ is defined as the steady state ratio. The time constant is obtained as

$$
\tau=\frac{M+K T_{d}}{K+C} .
$$

The first controller was chosen to minimize the error while remaining stable with $S_{r}=0.99$ and $\tau=0.15$, leading to $K=50000$ and $T_{d}=0.14$. The second controller was chosen to be less reactive to high frequency inputs with $S_{r}=0.55$ and $\tau=0.82$, leading to $K=500$ and $T_{d}=0.484$. In order for the system with the adaptive controller to remain so stable, the system represented in eqn. (9) must minimally be stable for any $K$ and $T_{d}$ obtainted from the adaptive controller law [35]. The adaptive controller linearly modifies the parameters between the first controller (when the index $V$ is below $5\left(V_{\text {min }}\right)$ ) 335 and the second controller (when the index $V$ is over $\left.15\left(V_{\max }\right)\right)$ and always leads to stable systems. As a general rule, the parameters should not be modified too quickly in order to avoid exciting the system.

Figure 10 shows the measured velocity with different control parameters for the force profile input shown in Fig. 8. With high control gains (first controller), the system reaches high velocities but is very reactive to high-frequency inputs. With low control gains (second controller), the system only reaches small velocities and would require large forces to move but is not very reactive to highfrequency inputs. Using the vibration controller, the system reaches high velocities and is not very reactive to high-frequency inputs.

\subsection{Parameters summary}

11

\section{Prototypes}

This section provides a brief description of the prototypes used for the experimental validation of the algorithms. 


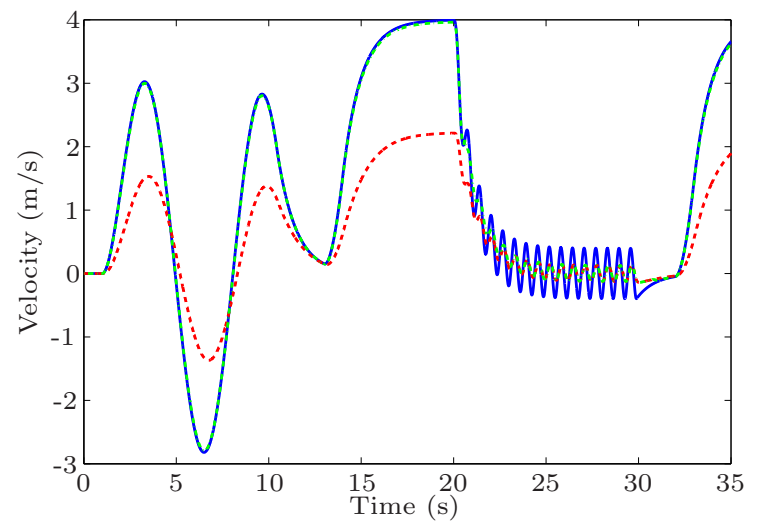

Figure 10: Velocity resulting from the force input shown in Fig. 8 The solid blue line represents the desired velocity and also the measured velocity obtained with high control gains (the two curves are virtually superimposed). The red dashed line is the velocity with low control gains. The green dashed-dot line is the velocity with the vibration controller (the control parameters are adapted between the high and low control gains)

Table 1: Suggested parameter values

\begin{tabular}{|l|l|}
\hline Parameter & \multicolumn{1}{c|}{ Value } \\
\hline$T_{w}$ & $3 / f_{l}$ \\
\hline$T_{n}$ & $1 /\left(2 f_{h}\right)$ \\
\hline$\lambda$ & 20 \\
\hline$L_{r}$ & $T_{w} \lambda$ \\
\hline$L_{f}$ & $T_{w} \cdot \lambda / 20$ \\
\hline$V_{\min }$ & $\lambda / 2$ \\
\hline$V_{\max }$ & $V_{\min }+\lambda$ \\
\hline$\eta_{\min }$ & 0.02 \\
\hline
\end{tabular}

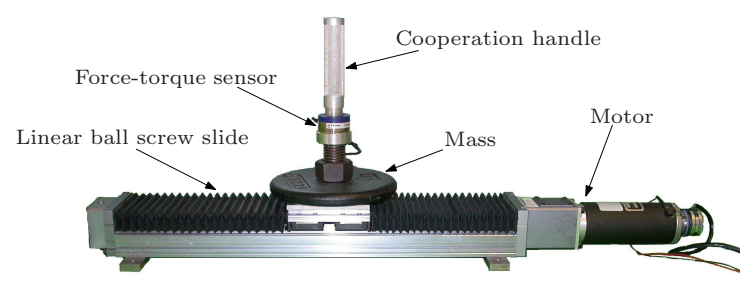

Figure 11: One DOF linear ball screw slide prototype.

\subsection{One-DOF linear ball screw slider}

The one-DOF linear ball screw slider is shown in Fig. 11. An ATI Mini-40 6-axis force/torque sensor is mounted between the end-effector and the han-
360

365

370 dle. Only the force component along the direction of motion of the slider is used for the experiments. The controller is implemented on a real-time QNX computer with a sampling period of $2 \mathrm{~ms}$ which is enough high for avoiding instability coming from sampling during the experiment. The algorithms are programmed using Simulink/RT-LAB software.

\subsection{Prototype of a 4-DOF intelligent assist device}

The robot used for the second series of experiments reported in this paper is a prototype of a 4-dof intelligent assist device (IAD) 1, shown in Fig. 12 This device allows three translations $(X Y Z)$ and a rotation $(\theta)$ about the vertical axis. In this prototype, the total moving mass is approximately $500 \mathrm{~kg}$ in the direction of the $X$ axis and $325 \mathrm{~kg}$ along the $Y$ axis. Additionally, the payload may vary between 0 and $113 \mathrm{~kg}$. The horizontal workspace is $3.3 m \times 2.15 m$ while the vertical range of motion is $0.52 \mathrm{~m}$. The range of rotation about the vertical axis is $120^{\circ}$. These characteristics are summarized in Tab. 2. As the first prototype, the controller is implemented on a real-time QNX computer with a sampling period of $2 \mathrm{~ms}$. The algorithms are programmed using Simulink/RT-LAB software.

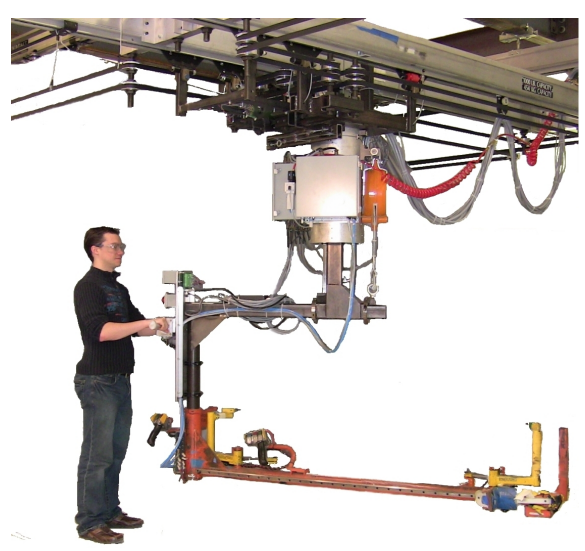

Figure 12: Prototype of a 4-dof intelligent assist device.

\section{Experimental validation}

This section presents the experiments performed with the two prototypes described in the preceding section. In these experiments, velocity control 
Table 2: Characteristics of the prototype of a 4-dof intelligent assist device.

\begin{tabular}{|c|c|c|c|c|}
\hline & $X$ axis & $Y$ axis & $Z$ axis & $\theta$ axis \\
\hline Range & $3.3 \mathrm{~m}$ & $2.15 \mathrm{~m}$ & $0.52 \mathrm{~m}$ & $120^{\circ}$ \\
\hline Moving inertia & $500 \mathrm{~kg}$ & $325 \mathrm{~kg}$ & $148 \mathrm{~kg}$ & $66 \mathrm{kgm}_{20}^{2}$ \\
\hline
\end{tabular}

is used with a proportional controller and friction compensation. No derivative gain is used since the signal is noisy (second derivative of the position) and no integral gain is used since the behaviour to a human input would then depend on the error history [23].

A control ratio, $\eta$, is used to adapt the control gains and is defined as

$$
\eta= \begin{cases}\eta_{\min } & \text { if } \eta^{\prime}<\eta_{\min } \\ \eta^{\prime} & \text { otherwise }\end{cases}
$$

where

$$
\eta^{\prime}= \begin{cases}1 & \text { if } V \leq V_{\min } \\ 0 & \text { if } V \geq V_{\max } \\ \frac{V_{\max }-V}{V_{\max }-V_{\min }} & \text { otherwise }\end{cases}
$$

and where $V_{\min }$ and $V_{\max }$ are respectively minimum and maximum index values and $\eta_{\min }$ is the minimum control ratio value.

Both the proportional gain and the friction compensation output are multiplied by the control ratio $\eta$, namely

$$
K=K^{*} \eta
$$

for the proportional controller and similarly for the friction compensation, where $K^{*}$ is the default proportional gain and $K$ is the actual proportional gain. From Fig. 7 and eqn. (9), it is readily observed that the system is of the second order, having two real poles, and that it remains stable for $\eta>0 . \quad V_{\min }$

When using a proportional controller, the adaptive law is fairly simple. With a more complex control algorithm, a design parameter (controller frequency for instance) could be simply multiplied by the control ratio $\eta$. An exemple of an adaptive law with a PD controller was shown in section 5.3 .

\subsection{One-DOF linear ball screw slider}

The experiments were performed with the following parameters. Virtual mass $m$ : $2 \mathrm{~kg}$, Virtual damping c: $60 \mathrm{Ns} / \mathrm{m}$, Default Proportional gain $K^{*}: 0.01$, Wide time window $T_{w}: 0.25 \mathrm{~s}$, Narrow time window $T_{n}: 0.03 \mathrm{~s}$, Rising Limit : $12.5 \mathrm{~s}^{-1}$, Falling Limit : $0.5 s^{-1}$, Minimum control ratio $\eta_{\min }$ : 0.02 , Minimum index value $V_{\min }: 17$, Maximum index value $V_{\max }: 34$, Scaling factor $\lambda: 20$.

Figure 13 shows an example of a low frequency interaction. This test was repeated using the vibration observer-controller and the results are the same with or without the controller. Indeed, no vibrations are detected and the vibration controller is thus not activated.

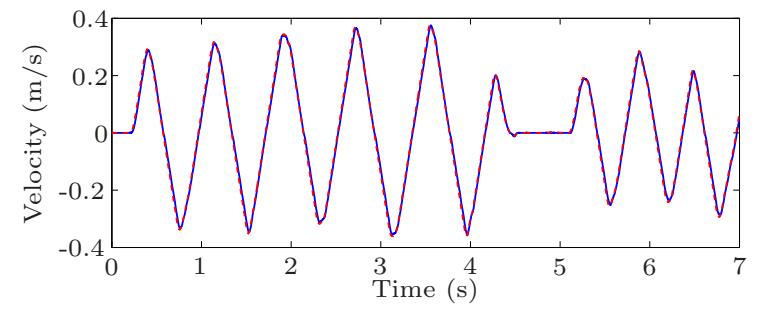

Figure 13: Low frequency interaction. The solid blue line is the measured velocity while the red dashed line is the desired velocity.

Figure 14 shows an example of interactive experiment without the vibration observer-controller. The operator's intention was to move the device at approximately $0.2 \mathrm{~m} / \mathrm{s}$ (which is a slow human arm speed) while being stiff. Both the desired and measured velocity include significant vibrations and the interaction is very uncomfortable.

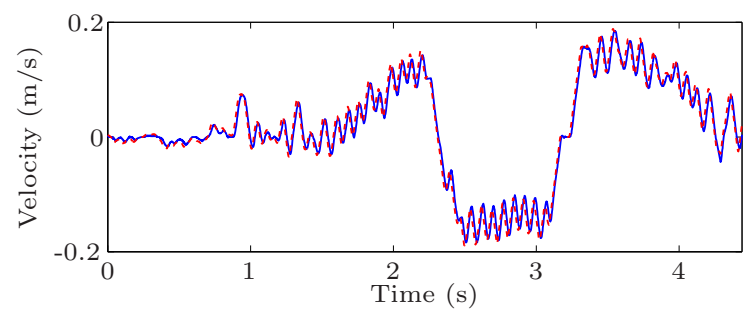

Figure 14: Stiff interaction without vibration observercontroller. The solid blue line is the measured velocity while the red dashed line is the desired velocity. This is not an unstable behaviour but a marginally stable situation which cannot be solve by using $\mathrm{PO} / \mathrm{PC}$.

Figure 15 shows an example using the vibration observer-controller. The intention of the human operator was to stay at a given position while being stiff and trying to minimally perturb the system. It can be observed that after one oscillation, after a very short period of time, the device is stabilized. 


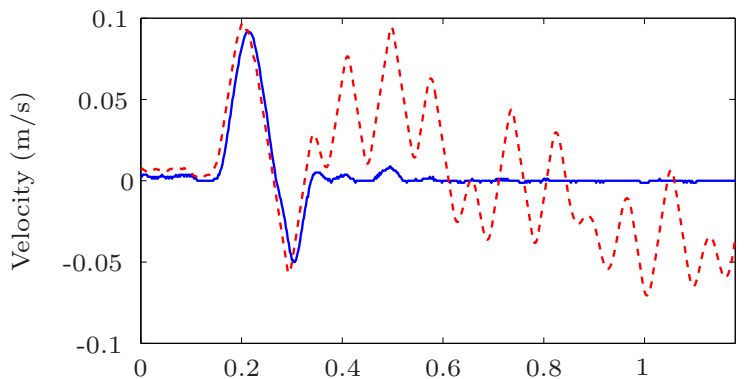

Figure 16 shows another example with the vibration observer-controller. The intention of the operator was to move the device at approximately $0.5 \mathrm{~m} / \mathrm{s}$ (which is a moderate human arm speed) while being stiff. The interaction is very stable and there is no vibration. The same interaction without vibration controller leads to significant vibrations. However, in this case, as soon as vibrations are detected, the control gains are reduced. The device's velocity does not reach the desired velocity but the interaction is smooth and safe.
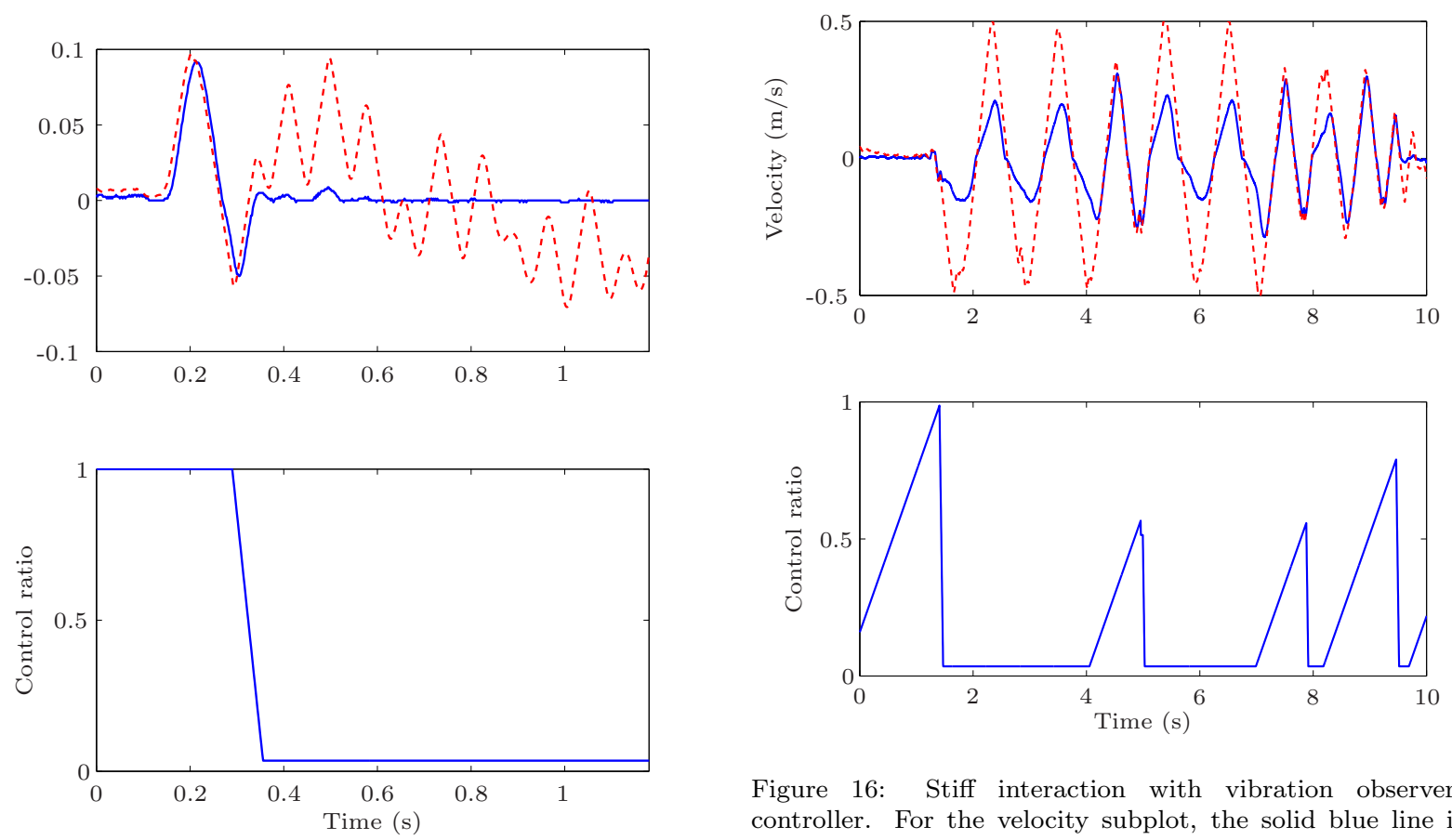

Figure 15: Stiff interaction around a given point with the vibration observer-controller. For the velocity subplot, the solid blue line is the measured velocity while the red dashed line is the desired velocity. The control ratio, equal to $\eta$, is the attenuation gain applied to the proportional controller and friction compensation output.

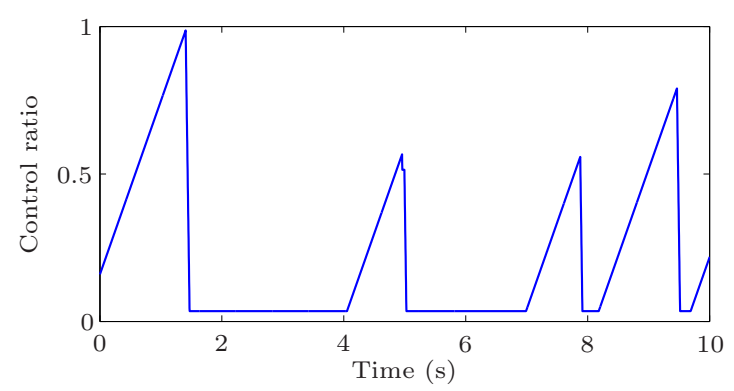

Figure 16: Stiff interaction with vibration observercontroller. For the velocity subplot, the solid blue line is the measured velocity while the red dashed line is the desired velocity.

\subsubsection{Passivity observer}

The data obtained from the experiment shown in Fig. 14 was also monitored with a passivity observer (PO) [11] and Fig. 17] shows the PO output (energy). This kind of observer has been used succesfully in many applications. However, it can be observed here that the energy does not become negative. In other words, the passivity controller (PC) does not detect any problem and would not apply any correction since it would not be activated. Indeed, the PO detects extra energy injected into the system by non-passive behaviour while it does not detect vibrations that are due to a stiff but passive environment. 


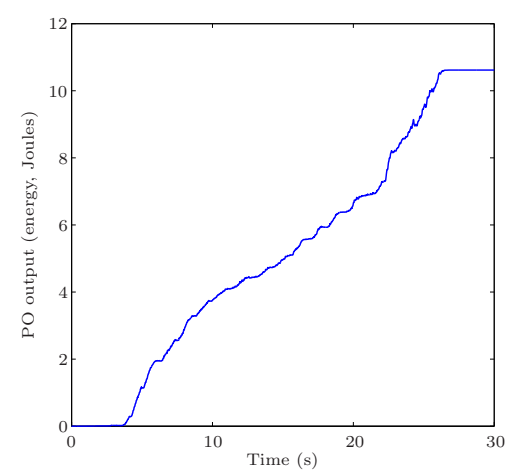

Figure 17: Passivity observer output with a stiff environment.

\subsection{4-DOF intelligent assist device}

Experiments were also performed with a 4-DOF Intelligent Assist Device (only one axis $(Y)$ is reported for simplicity). This experiment provides a demonstration of the algorithm's effectiveness on a different system which is also of a very different scale. The following parameters were used in the experiment. Virtual mass $m: 9 \mathrm{~kg}$, Virtual damping $c$ : $30 \mathrm{Ns} / \mathrm{m}$, Default Proportional gain $K^{*}: 0.06$, Wide time window $T_{w}: 1 \mathrm{~s}$, Narrow time window $T_{n}: 0.25 \mathrm{~s}$, Rising Limit : $12.5 \mathrm{~s}^{-1}$, Falling Limit : $0.5 s^{-1}$, Minimum control ratio $\eta_{\min }: 0.02$, Minimum index value $V_{\min }$ : 3 , Maximum index value $V_{\max }: 20$, Scaling factor $\lambda: 20$.

Figure 18 shows an example of a stiff interaction without the vibration observer-controller. Both the desired and measured velocity include significant vibrations and the interaction is very uncomfortable. Figure 19 shows an example of stiff interaction with the vibration observer-controller. The intention of the human operator was to stay at a given position while trying to minimally perturb the system. It can be observed that the device was stabilized.

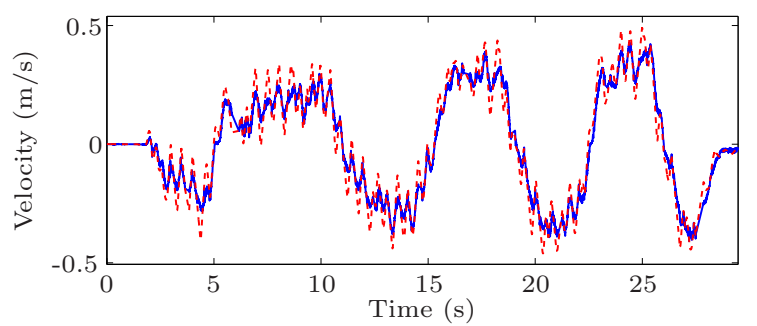

Figure 18: Stiff interaction without the vibration observercontroller. The solid blue line is the measured velocity while the red dashed line is the desired velocity.
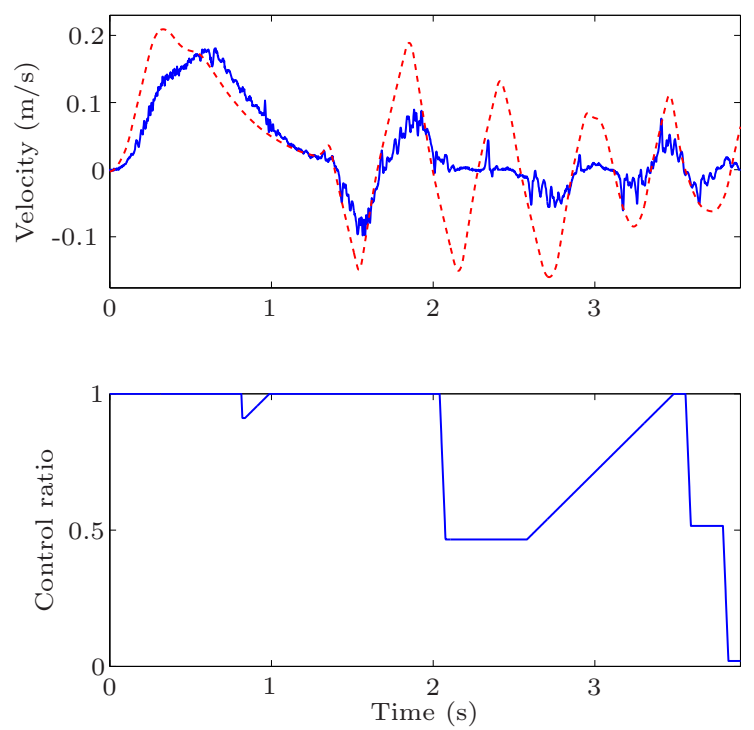

Figure 19: Stiff interaction around a point with the vibration observer-controller. For the velocity subplot, the solid blue line is the measured velocity while the red dashed line is the desired velocity. The control ratio, equal to $\eta$, is the attenuation gain applied to the proportional controller and to the friction compensation output.

\section{Discussion}

The algorithm was implemented on two prototypes. When interacting with a stiff environment, it has been shown that vibrations are very significant. By using the vibration observer and controller, the device follows the operator's intentions while eliminating or reducing vibrations. The trade-off is to decrease the task performance (the measured velocity does not reach the amplitude of the desired velocity for instance). However, the vibration controller is only activated when the vibrations are above a given treshold (both in frequency and amplitude) and therefore it does not influence the per-

\section{Conclusion}

In this paper a time-domain vibration observer and controller applied to physical Human-Robot Interaction (pHRI) was proposed. An observer detecting minima and maxima from a given signal with robustness in regards to noise was first presented. A vibration index based on these extrema was then defined. This index was then used by an adaptive controller in order to reduce the 
two different prototypes to demonstrate its performance. Future studies will focus on adaptive control laws for more complex controllers and on stability proofs.

\section{Acknowledgements}

This work was supported by The Natural Sciences and Engineering Research Council of Canada (NSERC) as well as by General Motors (GM) of Canada.

\section{References}

[1] C. Gosselin, T. Laliberte, B. Mayer-St-Onge, S. Foucault, A. Lecours, V. Duchaine, N. Paradis, D. Gao R. Menassa, A friendly beast of burden: A humanassistive robot for handling large payloads, IEEE Robotics Automation Magazine 20 (4) (2013) 139-147. doi: 10.1109/MRA.2013.2283651

[2] J. E. Colgate, M. Peshkin, S. H. Klostermeyer, Intelligent assist devices in industrial applications: a review, in: Proceedings of the International Conference 2521. doi:10.1109/IROS.2003.1249248

[3] R. Bischoff, J. Kurth, G. Schreiber, R. Koeppe, A. Albu-Schaeffer, A. Beyer, O. Eiberger, S. Haddadin, A. Stemmer, G. Grunwald, G. Hirzinger, The KUKADLR lightweight robot arm - a new reference platform for robotics research and manufacturing, 41st International Symposium on Robotics (2010) $1-8$.

[4] E. Guizzo, E. Ackerman, The rise of the robot worker, IEEE Spectrum 49 (10) (2012) 34-41. doi:10.1109/ MSPEC. 2012.6309254

[5] http://www.universal-robots.com/ visited May 02, 2014.

[6] A. Mörtl, M. Lawitzky, A. Kucukyilmaz, M. Sezgin, C. Basdogan, S. Hirche, The role of roles: Physical cooperation between humans and robots, The International Journal of Robotics Research.

[7] Y. Li, K. P. Tee, W. L. Chan, R. Yan, Y. Chua, D. Limbu, Continuous role adaptation for human robot shared control, Robotics, IEEE Transactions on 31 (3) (2015) 672-681.

[8] M. Erden, A. Billard, Hand impedance measurements during interactive manual welding with a robot, IEEE Transactions on Robotics 31 (1) (2015) 168-179.

[9] R. Adams, B. Hannaford, Stable haptic interaction with virtual environments, IEEE Transactions on Robotics and Automation 15 (3) (1999) 465 - 74.

[10] J. Gil, A. Avello, A. Rubio, J. Florez, Stability analysis of a 1 dof haptic interface using the routh-hurwitz criterion, IEEE Transactions on Control Systems Technology 12 (4) (2004) 583-588. doi:10.1109/TCST . 2004. 825134

[11] B. Hannaford, J.-H. Ryu, Time-domain passivity control of haptic interfaces, Transactions on Robotics and Automation 18 (1) (2002) 1 - 10. doi:10.1109/70. 988969.

[12] J.-H. Ryu, D.-S. Kwon, B. Hannaford, Stability guaranteed control: Time domain passivity approach, Transactions on Control Systems Technology 12 (6) (2004) 860 - 868. doi:10.1109/TCST.2004.833648 15] H. C. Cho, J. H. Park, Impedance control with variable damping for bilateral teleoperation under time delay, JSME International Journal, Series C: Mechanical Systems, Machine Elements and Manufacturing 48 (4) (2006) 695 - 703. doi:10.1299/jsmec.48.695

585 [16] B. Hannaford, J.-H. Ryu, Time domain passivity control of haptic interfaces, in: IEEE International Conference on Robotics and Automation, Vol. 2, 2001, pp. 1863 - 1869. doi:10.1109/70.988969

[17] S. Hirche, M. Buss, Passive position controlled telepresence systems with time delay, in: American Control Conference, Vol. 1, Denver, CO, United States, 2003, pp. $168-173$.

[18] M. Fardad, B. Bamieh, A frequency domain analysis and synthesis of the passivity of sampled-data systems, in: IEEE Conference on Decision and Control, Vol. 3, Nassau, Bahamas, 2004, pp. 2358 - 2363. doi:10.1109/ CDC.2004.1428749

[19] D. Ryu, J.-B. Song, S. Kang, M. Kim, Frequency domain stability observer and active damping control for stable haptic interaction, Control Theory Applications, IET 2 (4) (2008) 261 -268. doi:10.1109/ROBOT. 2007. 363772

[20] C. Carignan, K. Cleary, Closed-loop force control for haptic simulation of virtual environments, Haptics-e 1 (2) (2000) $1-14$.

[21] V. Hayward, K. Maclean, Do it yourself haptics: part i, IEEE Robotics Automation Magazine 14 (4) (2007) 88 -104. doi:10.1109/M-RA.2007.907921

[22] R. v. d. Linde, Hapticmaster - a generic force controlled robot for human interaction, Industrial Robot: An International Journal 30 (2003) 515-524. doi:doi: 10.1108/01439910310506783

[23] A. Lecours, B. Mayer-St-Onge, C. Gosselin, Variable admittance control of a four-degree-of-freedom intelligent assist device, in: IEEE International Conference on Robotics and Automation, Saint Paul, USA, 2012, pp. 3903-3908. doi:10.1109/ICRA.2012.6224586

[24] K. Kosuge, N. Kazamura, Control of a robot handling an object in cooperation with a human, in: 6th IEEE International Workshop RO-MAN Proceedings., 1997, pp. $142-147$. doi:10.1109/ROMAN.1997.646971

[25] A. Lecours, C. Gosselin, Computed-torque control of a four-degree-of-freedom admittance controlled intelligent assist device, in: Experimental Robotics, Vol. 88 of Springer Tracts in Advanced Robotics, Springer International Publishing, 2013, pp. 635-649.

[26] R. Ikeura, H. Inooka, Variable impedance control of a robot for cooperation with a human, in: Proceedings of the IEEE International Conference on Robotics and Automation, Vol. 3, 1995, pp. 3097-3102. doi:10.1109/ 
ROBOT.1995.525725

[27] T. Tsumugiwa, R. Yokogawa, K. Hara, Variable impedance control with regard to working process for man-machine cooperation-work system, in: Proceedings of the IEEE/RSJ International Conference on Intelligent Robots and Systems, Vol. 3, 2001, pp. 15641569. doi:10.1109/IROS.2001.977202

[28] H. T. Jung, Seul, Stability and convergence analysis of robust adaptive force tracking impedance control of 640 robot manipulators, Vol. 2, 1999, pp. 635-640. doi: 10.1109/IROS.1999.812751

[29] H. A. Zeng, G., An overview of robot force control, Robotica 15 (5) (1997) 473-482.

[30] D. Surdilovic, Robust control design of impedance control for industrial robots, in: IEEE International Conference on Intelligent Robots and Systems, 2007, pp. 3572-3579. doi:10.1109/IROS.2007.4399456

[31] H. A.-C. Chien, M.-C., Adaptive impedance control of robot manipulators based on function approximation technique, Robotica 22 (4) (2004) 395-403. doi:10. 1017/S0263574704000190

[32] T. Tsumugiwa, R. Yokogawa, K. Yoshida, Stability analysis for impedance control of robot for human-robot cooperative task system, in: International Conference on Intelligent Robots and Systems, Vol. 4, Sendai,

口 Japan, 2004, pp. 3883 - 8. doi:10.1109/IROS.2004. 1390020

[33] V. Duchaine, C. Gosselin, Investigation of human-robot interaction stability using lyapunov theory, in: IEEE International Conference on Robotics and Automation, 2008, pp. 2189 -2194. doi:10.1109/ROBOT.2008. 4543531

[34] A. Ditkowski, A. Bhandari, B. Sheldon, Computing derivatives of noisy signals using orthogonal functions expansions, Journal of Scientific Computing 36 (3) (2008) 333-349. doi:10.1007/s10915-008-9193-9

[35] D. Liberzon, A. Morse, Basic problems in stability and design of switched systems, IEEE Control Systems 19 (5) (1999) 59-70. doi:10.1109/37.793443 